\title{
Performance, rumen development, and carcass traits of male calves fed starter concentrate with crude glycerin
}

\section{Raylon Pereira Maciel ${ }^{1}$, José Neuman Miranda Neiva ${ }^{2}$, João Restle ${ }^{2}$, Ubirajara Oliveira Bilego ${ }^{3}$, Fabrícia Rocha Chaves Miotto ${ }^{2}$, Antônio João Fontes ${ }^{4}$, Maria Clorinda Soares Fiovaranti ${ }^{5}$, Rhaiza Alves de Oliveira ${ }^{2}$}

\author{
${ }^{1}$ Universidade Federal Rural da Amazônia, Campus de Parauapebas, PA, Brazil. \\ ${ }^{2}$ Universidade Federal do Tocantins, Escola de Medicina Veterinária e Zootecnia, Programa de Pós-graduação em Ciência Animal Tropical, \\ Araguaína, TO, Brazil. \\ ${ }^{3}$ Cooperativa Agroindustrial dos Produtores Rurais do Sudeste Goiano, Rio Verde, GO, Brazil. \\ ${ }^{4}$ Instituto Federal Goiano, Rio Verde, GO, Brazil. \\ ${ }^{5}$ Universidade Federal de Goiás, Departamento de Medicina Veterinária, Goiânia, GO, Brazil.
}

\begin{abstract}
The objective of this study was to assess the effects of including crude glycerin in the diet on intake, performance, rumen development, and carcass traits of dairy crossbred veal calves fed starter concentrate containing 0,80 , 160 , and $240 \mathrm{~g} \mathrm{~kg}^{-1}$ crude glycerin. Twenty-eight calves with an average weight of $38.03 \pm 6.7 \mathrm{~kg}$ and five days of age were distributed in a completely randomized design with four treatments with seven replications. Calves were individually housed in covered stalls equipped with feeders and drinkers for 56 days. The calf response to inclusion of crude glycerin in the concentrate changed over the weeks and the inclusion level of $240 \mathrm{~g} \mathrm{~kg}^{-1}$ resulted in greater dry matter intake and average daily gain. There was no effect on the final weight and total weight gain of the animals, with mean values of 73.60 and $35.16 \mathrm{~kg}$, respectively. The weight of the rumen-reticulum adjusted for body weight, empty body weight, and total stomach weight increased linearly with the inclusion of crude glycerin. Blood total protein, globulin, urea, cholesterol, gamma glutamyl transferase, aspartate aminotransferase, and alkaline phosphatase concentrations did not differ among treatments. Carcass traits and meat color were not affected. Crude glycerin can be added to dairy calf starter concentrate up to $240 \mathrm{~g} \mathrm{~kg}^{-1} \mathrm{dry}_{\text {matter because it benefits }}$ concentrate intake, performance, and rumen development without affecting animal health.
\end{abstract}

Key Words: biofuel, byproducts, veal calves, weight gain

\section{Introduction}

Using adequate feeding and management techniques when raising male dairy veal calves allows these animals to be exploited for quality meat production and improves the efficiency of dairy farms. However, the high cost of the initial phase of rearing is one of the limiting factors for the expansion and consolidation of these animals for meat production in Brazil. Supplying solid food from the first weeks of life is the main way to stimulate the transition of the newborn animal to the condition of ruminant, accelerate rumen development, and decrease weaning age.

Using by-products in the starter concentrate to substitute noble ingredients, such as corn and soybean, can also be an alternative strategy for economic exploitation

Received July 2, 2015 and accepted October 26, 2015

Corresponding author: raylonmaciel@gmail.com

http://dx.doi.org/10.1590/S1806-92902016000600005

Copyright (C) 2016 Sociedade Brasileira de Zootecnia. This is an Open Access article distributed under the terms of the Creative Commons Attribution License (http://creativecommons.org/licenses/by/4.0/), which permits unrestricted use, distribution, and reproduction in any medium, provided the original work is properly cited. of these animals to reduce rearing costs without impairing the animal performance. Crude glycerin is a by-product derived from oil and fat transesterification that is generated in approximately $10 \%$ of biofuel production (Rahmat et al., 2010) and has aroused great interest as a food alternative for feeding ruminants due to its energetic value that is similar to that of corn (Donkin, 2008).

Information relating crude glycerin in the development of the rumen for young calves is not known, but its use as a feed additive has been reported. According to Napoles et al. (2012), crude glycerin can be used as an alternative energy source in substitution of corn in calf starter concentrate up to $100 \mathrm{~g} \mathrm{~kg}^{-1}$ of the DM without affecting ruminal or blood parameters. Adding $150 \mathrm{~g} \mathrm{~kg}^{-1}$ crude glycerin to dried milk did not affect the performance or health of Friesian calves (Drackley, 2008). The crude glycerin can replace up to $460 \mathrm{~g} \mathrm{~kg}^{-1}$ of the total lactose in milk replacers (Ebert et al., 2008; Raeth-Knight et al., 2009) or it can be used as a component of oral rehydration solution for young calves without any impacts on calf performance or health (Werner Omazic et al., 2013). Regarding carcass traits or chemical composition of the 
longissimus lumborum area, no significant effects were reported when crude glycerin was included at $300 \mathrm{~g} \mathrm{~kg}^{-1}$ DM in the finishing diet of steers (Mach et al., 2009; Bartoň et al., 2013; Van Cleef et al., 2014).

The objective of the present study was to assess the effect of including crude glycerin in the starter concentrate on performance, rumen development, blood parameters, and carcass traits of crossbred dairy calves.

\section{Material and Methods}

All the procedures and protocols used in the present experiment were approved by the Committee of Ethics on Animal Use of Universidade Federal do Tocantins (CEUAUFT), under protocol no. 23101.003936/2012-00.

The experiment was carried out in Rio Verde - GO, Brazil, from February to April 2011. Thirty-five crossbred Friesian-Zebu feeder calves with an average body weight $38.03 \pm 7.2 \mathrm{~kg}$ and average age of five days were used. Seven of them were slaughtered at the start of the experiment and used as reference to calculate carcass gain, and 28 were placed in a completely randomized design with four treatments: 0 , 80,160 , and $240 \mathrm{~g} \mathrm{~kg}^{-1}$ dry matter (DM) of crude glycerin in the starter concentrate, with seven replications to assess the animal performance. The concentrate was formulated according to the recommendations of NRC (2001) to meet the nutritional needs of unweaned calves (Table 1). The crude glycerin (GENPA ${ }^{\circledR}-80$ - nutritional energetic glycerol for feeding) used to make up the diets came from soybean oil and contained $899.8 \mathrm{~g} \mathrm{~kg}^{-1}$ dry matter, $11.9 \mathrm{~g} \mathrm{~kg}^{-1} \mathrm{DM}$ ether extract, $78.6 \mathrm{~g} \mathrm{~kg}^{-1} \mathrm{DM}$ mineral matter, $803.5 \mathrm{~g} \mathrm{~kg}^{-1}$ DM glycerol, $74.7 \mathrm{~g} \mathrm{~kg}^{-1} \mathrm{DM}$ sodium chloride, less than $0.1 \mathrm{~g} \mathrm{~kg}^{-1} \mathrm{DM}$ methanol, and $1.27 \mathrm{~g} \mathrm{~cm}^{-3}$ density.

The animals were identified with earrings, restrained by ropes and a collar in individual, movable, covered stalls. Each calf received $4 \mathrm{~L}$ whole milk day ${ }^{-1}$, divided into two daily meals, at $08.00 \mathrm{~h}$ and $16.00 \mathrm{~h}$, supplied at $38^{\circ} \mathrm{C}$ in individual buckets. After the fifth day, they also received starter concentrate freely, and the quantity supplied and the orts from each animal were recorded daily to estimate the dry matter and nutrient intakes. Animals had access to fresh water ad libitum.

The animals were weighed on the fifth day of life, which was when the experiment started, and then weekly, on electronic scales, always in the morning before the diet was supplied until the eighth week of life, when the experimental period ended. At the time of the weighing, the hip and shoulder heights, hip width, body length, and thoracic girth were measured using a ruler and a tape measure.
Samples of concentrate supplied and orts were collected weekly. All these samples were pre-dried in a forced-air oven at $55{ }^{\circ} \mathrm{C}$ for $72 \mathrm{~h}$, ground in a grinder with $1 \mathrm{~mm}$ mesh and stored for later analysis. The food samples and orts were analyzed for the contents of dry matter, organic matter, crude protein, neutral detergent insoluble nitrogen, acid detergent insoluble nitrogen, lignin, and ash according to the Association of Official Agricultural Chemists (AOAC, 1995) and neutral detergent fiber (NDF), acid detergent fiber (ADF), and ether extract according to the methodology recommended by the manufacturer of the ANKON apparatus. For the NDF and ADF analysis, $4 \times 5 \mathrm{~cm}$ nonwoven fabric bags (TNT $\left.-100 \mathrm{~g} / \mathrm{cm}^{2}\right)$ were used. The non-fibrous carbohydrates (NFC) were calculated using the following equation (Sniffen et al., 1992): $\mathrm{NFC}=100$ $-(\% \mathrm{NDF}+\% \mathrm{CP}+\% \mathrm{EE}+\%$ ash $)$. The total digestible nutrient content (TDN) was calculated following the prediction equation used by the NRC (2001).

Ten milliliters of blood were collected on the first, fourth, and eighth weeks of life by jugular puncture using vacuum tubes (Vacutainer ${ }^{\circledR}$ ) with a lid and potassium EDTA as anticoagulant. The samples were centrifuged at $2000 \times \mathrm{g}$ for $20 \mathrm{~min}$ to obtain the serum. The serum was then separated by aspiration, divided into aliquots and placed in plastic tubes with a lid $\left(\right.$ Eppendorf $\left.^{\circledR}\right)$, labelled

Table 1 - Proportions of ingredients and chemical composition of experimental diets

\begin{tabular}{lcccc}
\hline & \multicolumn{4}{c}{ Crude glycerin $\left(\mathrm{g} \mathrm{kg}^{-1} \mathrm{DM}\right)$} \\
\cline { 2 - 5 } & 0 & 80 & 160 & 240 \\
\hline Ingredient & & & & \\
Ground corn grain & 743.8 & 647.5 & 550.0 & 452.5 \\
Soybean meal & 226.2 & 242.5 & 260.0 & 277.5 \\
Crude glycerin & - & 80.0 & 160.0 & 240.0 \\
Coopergold ${ }^{\circledR} 3^{1}$ & 30.0 & 30.0 & 30.0 & 30.0 \\
Nutrient (g kg ${ }^{-1}$ DM) & & & & \\
Dry matter & 849.4 & 831.1 & 808.2 & 806.8 \\
Crude protein & 181.0 & 183.7 & 182.4 & 182.6 \\
Neutral detergent fiber & 100.3 & 104.6 & 104.8 & 100.8 \\
Acid detergent fiber & 35.7 & 34.7 & 34.6 & 34.9 \\
Non-fiber carbohydrates & 651.8 & 632.2 & 618.2 & 611.2 \\
Ether extract & 18.2 & 19.8 & 25.7 & 29.0 \\
Total digestible nutrients & & & & \\
NDIN (\% N total) & 806.2 & 790.0 & 780.4 & 786.8 \\
ADIN (\% N total) & 10.11 & 17.27 & 26.90 & 22.45 \\
Lignin & 4.09 & 3.02 & 2.72 & 2.70 \\
Ash & 10.71 & 9.92 & 9.29 & 8.35 \\
\hline
\end{tabular}

DM - dry matter; NDIN - neutral detergent insoluble nitrogen; ADIN - acid detergent insoluble nitrogen.

${ }^{1} \mathrm{Ca}-22 \mathrm{~g} \mathrm{~kg}^{-1} ; \mathrm{P}-9 \mathrm{~g} \mathrm{~kg}^{-1} ; \mathrm{Na}-4.5 \mathrm{~g} \mathrm{~kg}^{-1}$ (only for the diet without crude glycerin); $\mathrm{K}-0.08 \mathrm{~g} \mathrm{~kg}^{-1} ; \mathrm{Mg}-2.00 \mathrm{~g} \mathrm{~kg}^{-1} ; \mathrm{S}-3.75 \mathrm{~g} \mathrm{~kg}^{-1} ; \mathrm{Fe}-4673.20 \mathrm{mg} \mathrm{kg}^{-1}$; vit. A - $333.40 \mathrm{IU} \mathrm{g}^{-1}$; vit. D3 - $100.02 \mathrm{IU} \mathrm{g}^{-1}$; vit. E - $333.4 \mathrm{mg} \mathrm{kg}^{-1}$; saccharomyces cerevisea C. $1026-833.00 \mathrm{col} \mathrm{kg}{ }^{-1}$; Fe ORG - $666.60 \mathrm{mg} \mathrm{kg}^{-1}$; Cu ORG - $0.18 \mathrm{mg} \mathrm{kg}{ }^{-1}$; Mn ORG - $0.39 \mathrm{mg} \mathrm{kg}^{-1} ; \mathrm{Zn} \mathrm{ORG} \mathrm{-} 333.40 \mathrm{mg} \mathrm{kg}^{-1}$; Se ORG - $3.28 \mathrm{mg} \mathrm{kg}^{-1}$; $\mathrm{Fe}-4671.95 \mathrm{mg} \mathrm{kg}^{-1} ; \mathrm{Cu}-833.40 \mathrm{mg} \mathrm{kg}^{-1} ; \mathrm{Mn}-2134.00 \mathrm{mg} \mathrm{kg}^{-1} ; \mathrm{Zn}-3334.00 \mathrm{mg} \mathrm{kg}^{-1}$; $\mathrm{Co}-20.00 \mathrm{mg} \mathrm{kg}^{-1}$; $-83.40 \mathrm{mg} \mathrm{kg}^{-1}$; $\mathrm{Se}-33.40 \mathrm{mg} \mathrm{kg}^{-1}$; monensin sodium $833.40 \mathrm{mg} \mathrm{kg}^{-1}$; biotin - $4125.00 \mathrm{MCG} \mathrm{kg}^{-1}$.

${ }^{2}$ Estimated by NRC equations (2001). 
and frozen in a freezer for later biochemical analysis. The serum biochemical analyses (cholesterol, total protein, urea, albumin, creatinine, aspartate aminotransferase, alkaline phosphatase, and gamma glutamyl transferase) were carried out at $37^{\circ} \mathrm{C}$, using commercial reagents. The reactions were read by a Bioplus ${ }^{\circledR}$ automatic biochemical analyzer (spectrophotometer) model Bio-2000 IL-A. The globulin concentration was calculated as the difference between total proteins and albumin.

At the eighth week, the animals were weighed and then slaughtered by brain concussion, followed by cutting the jugular and carotenoid veins according to Normative Instruction no. 3 of 01/13/2000 (Technical Regulation of Stunning Methods for Humane Slaughter of Butcher Animals).

For the morphometric assessment of the upper digestive tract, after slaughter, the abdominal cavity was opened, the four compartments (rumen-reticulum, omasum, and abomasum) were removed, and the contents of the tract were washed out with water. The volume of all the compartments was measured by tying closed the exits and filling them with water to maximum capacity, and then the volume was measured using a graded testtube. After removing the excess water from the tissues, the weight of the rumen-reticulum, omasum, abomasum, and the compartments together were measured. Samples of the cranial portion of the ventral coronary pillar in the rumen ventral sac were removed using a scalpel, preserved in $10 \%$ formaldehyde, and then the papillae height and width were assessed using a stereoscopic microscope and a millimeter ruler as proposed by Lesmeister et al. (2004).

After slaughter, the carcasses were weighed to obtain the hot carcass weight (HCW) and then placed in a cold room at 0 to $2{ }^{\circ} \mathrm{C}$ for $24 \mathrm{~h}$ and weighed again to obtain the cold carcass weight (CCW). The empty body weight (EBW) was obtained directly by summing the weights of the hooves, head, skin, blood, organs, viscera, internal fats, and carcass. In the right half carcass, a cut was made between the 12th and 13th ribs to expose the longissimus lumborum muscle. An outline of the area of this muscle was drawn on parchment paper and its area was measured and expressed in $\mathrm{cm}^{2}$ using the Image $J^{\circledR}$ program. The physical composition of the carcass was estimated using the methodology of Hankins and Howe (1946). In the left carcass, half of the primal pistol cut was separated from the forequarter between the fifth and sixth ribs, and from the flank by a cut $16 \mathrm{~cm}$ from the spine. The pistol cut was separated into commercial or secondary cuts (rump uk trim, tail of rump, striploin, eye of round, rump cap, inside, outside, shank, cuberoll, tenderloin, and knuckle) and each piece was weighed individually.
Before analysis, the assumptions of normal distribution and homoscedasticity were checked for all the variables. The initial age was used as co-variable, and when it was not significant, the effect was removed from the model. To assess the effect of the treatments, $\alpha=0.05$ was adopted, with the mathematical model represented by:

$$
\gamma_{\mathrm{ij}}=\mu+\tau_{\mathrm{i}}+£_{\mathrm{j}}+\tau_{\mathrm{i}}^{*} £_{\mathrm{j}}+\varepsilon_{\mathrm{ij}}
$$

in which $\gamma_{\mathrm{ij}}=$ dependent variable; $\mu=$ overall mean; $\tau_{\mathrm{i}}=$ effect of factor $\mathrm{i}$ (age); $£_{\mathrm{j}}=$ effect of factor $\mathrm{j}$ (crude glycerin); $\left(\tau_{\mathrm{i}}{ }^{*} £_{\mathrm{j}}\right)=$ interaction between factor $\mathrm{i}$ and factor $\mathrm{j}$; and $\varepsilon_{\mathrm{ij}}=$ residual experimental error. In the regression study, the model was:

$$
\gamma_{i j}=\beta 0+\beta 1 x i+\beta 2 x^{2}+\beta 3 x i^{3}+\alpha j+\varepsilon i j,
$$

in which $\gamma \mathrm{ij}=$ dependent variables; $\beta=$ regression coefficients; $x i=$ substitution levels; $\alpha j=$ deviations from the regression; and $\varepsilon \mathrm{ij}=$ random residual error.

\section{Results}

Dry matter intake was influenced by including crude glycerin in the feeder calf concentrate (Table 2), and a linear increase was observed as the calf age increased $(\mathrm{P}<0.01)$. Including crude glycerin in the concentrate altered the response of the calves over the weeks, and adding $240 \mathrm{~g} \mathrm{~kg}^{-1}$ provided the highest DMI (Figure 1). Crude protein intake and total digestible nutrients showed the same response as DMI.

Average daily gain (ADG) was affected by adding crude glycerin to the feeder calf concentrate (Table 2) and a linear increase was observed as the calves became older $(\mathrm{P}<0.001)$. Including crude glycerin in the concentrate altered the ADG over the weeks, and the level of $240 \mathrm{~g} \mathrm{~kg}^{-1}$ inclusion resulted in the highest $\mathrm{ADG}$ (Figure 2). There was no effect $(\mathrm{P}>0.05)$ of adding crude glycerin on the final weight, total weight gain of the animals, and feed

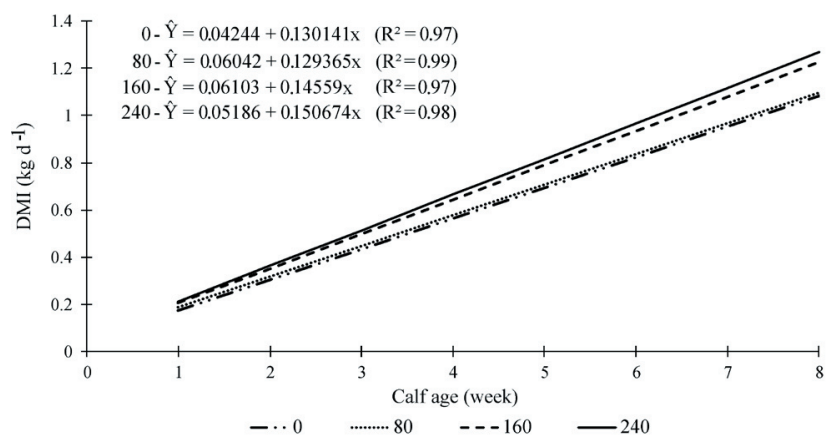

Figure 1 - Daily dry matter intake of feeder calves receiving starter concentrates containing crude glycerin over the experimental period. 
conversion (Table 3). Body measurements of the calves increased with age $(\mathrm{P}<0.01)$, but only the thoracic girth and hip width increased linearly $(\mathrm{P}<0.05)$, by 0.06 and $0.03 \mathrm{~cm}$, for each gram of crude glycerin added to the concentrate, respectively (Table 4).

Including crude glycerin in the concentrate did not alter $(\mathrm{P}>0.05)$ rumen-reticulum weight when expressed in absolute weight. However, when the weight of this component was adjusted to the BW, EBW, and total stomach weight, a linear increase was observed $(\mathrm{P}<0.05)$, of 0.0013 , 0.0011 , and 0.015 percentage points for each gram of crude glycerin added to the concentrate, respectively (Table 5). The rumen-reticulum volumes were not affected $(\mathrm{P}>0.05)$ by including crude glycerin (Table 5 ).

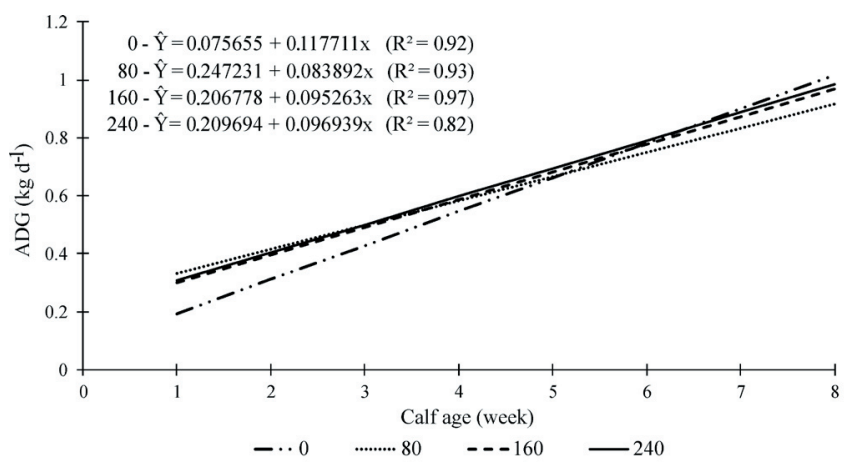

Figure 2 - Performance (average daily gain) of feeder calves receiving starter concentrate containing crude glycerin over the experimental period.

Table 2 - Performance variables of dairy feeder calves receiving starter concentrate with addition of crude glycerin

\begin{tabular}{|c|c|c|c|c|c|c|c|c|}
\hline \multirow{2}{*}{ Variable } & \multicolumn{4}{|c|}{ Crude glycerin ( $\left.\mathrm{g} \mathrm{kg}^{-1} \mathrm{DM}\right)$} & \multirow{2}{*}{ Mean $\pm \mathrm{SE}$} & \multicolumn{3}{|c|}{ P-value } \\
\hline & 0 & 80 & 160 & 240 & & CG & A & $\mathrm{CG} \times \mathrm{A}$ \\
\hline DMI, $\mathrm{kg} \mathrm{d}^{-1}$ & 0.425 & 0.461 & 0.473 & 0.518 & $0.469 \pm 0.08$ & 0.001 & 0.001 & 0.002 \\
\hline TDMI, $\mathrm{kg} \mathrm{d}^{-1}$ & 0.932 & 0.968 & 0.980 & 1.025 & $0.976 \pm 0.08$ & 0.001 & 0.001 & 0.002 \\
\hline TCPI, $\mathrm{kg} \mathrm{d}^{-1}$ & 0.207 & 0.216 & 0.217 & 0.226 & $0.217 \pm 0.01$ & 0.001 & 0.001 & 0.001 \\
\hline TDNI, $\mathrm{kg} \mathrm{d}^{-1}$ & 0.347 & 0.368 & 0.372 & 0.410 & $0.374 \pm 0.06$ & 0.001 & 0.001 & 0.01 \\
\hline $\mathrm{DMI} / \mathrm{BW}, \mathrm{g} \mathrm{kg}^{-1} \mathrm{~d}^{-1}$ & 7.27 & 7.63 & 8.19 & 8.91 & $8.08 \pm 0.37$ & 0.142 & 0.001 & 0.987 \\
\hline
\end{tabular}

SE - standard error; CG - crude glycerin; A - age.

Regression equations for the level of crude glycerin addition: DMI - dry matter intake $\left(\hat{\mathrm{Y}}=0.426+0.0004 \mathrm{x}, \mathrm{R}^{2}=0.96 ; \mathrm{DMI} / \mathrm{BW}-\mathrm{dry}\right.$ matter intake/body weight: $\hat{\mathrm{Y}}=0.731+$ $\left.0.0006 \mathrm{x}, \mathrm{R}^{2}=0.97\right)$; DMI, $\left.\mathrm{g} \mathrm{kg}^{-1} \mathrm{BW}^{0.75}: \hat{\mathrm{Y}}=19.86+0.018 \mathrm{x}, \mathrm{R}^{2}=0.98\right)$; TDMI - total dry matter intake (milk + concentrate: $\hat{\mathrm{Y}}=0.932+0.0003 \mathrm{x}, \mathrm{R}^{2}=0.96$ ); CPI - crude protein intake $\left(\hat{\mathrm{Y}}=0.076+0.00007 \mathrm{x}, \mathrm{R}^{2}=0.95\right)$; TCPI - total crude protein intake $\left(\hat{\mathrm{Y}}=0.207+0.00007 \mathrm{x}, \mathrm{R}^{2}=0.95\right)$; TDNI - total digestible nutrient intake $(\hat{\mathrm{Y}}=0.345+0.0002 \mathrm{x}$ $\left.\mathrm{R}^{2}=0.90\right) ; \mathrm{ADG}$ - average daily gain $\left(\hat{\mathrm{Y}}=0.608+0.0001 \mathrm{x}, \mathrm{R}^{2}=0.97\right)$.

Table 3 - Performance variables of dairy feeder calves receiving starter concentrate with addition of crude glycerin

\begin{tabular}{|c|c|c|c|c|c|c|c|}
\hline \multirow{2}{*}{ Variable } & \multicolumn{4}{|c|}{ Crude glycerin ( $\left.\mathrm{g} \mathrm{kg}^{-1} \mathrm{DM}\right)$} & \multirow{2}{*}{ Mean $\pm \mathrm{SE}$} & \multicolumn{2}{|c|}{ P-value } \\
\hline & 0 & 80 & 160 & 240 & & $\mathrm{~L}$ & Q \\
\hline Initial weight, $\mathrm{kg}$ & 39.03 & 39.13 & 36.84 & 38.76 & $38.03 \pm 7.2$ & - & - \\
\hline Final weight, $\mathrm{kg}$ & 72.93 & 74.11 & 72.43 & 74.93 & $73.60 \pm 11.67$ & 0.790 & 0.753 \\
\hline Total weight gain, $\mathrm{kg}$ & 33.90 & 34.99 & 35.59 & 36.17 & $35.16 \pm 7.09$ & 0.541 & 0.927 \\
\hline Feed conversion, $\mathrm{kg} \mathrm{DM} \mathrm{kg}^{-1} \mathrm{WG}$ & 0.7 & 0.72 & 0.72 & 0.80 & $0.73 \pm 0.34$ & 0.318 & 0.757 \\
\hline Total feed conversion, $\mathrm{kg} \mathrm{DM} \mathrm{kg}^{-1} \mathrm{WG}$ & 1.55 & 1.58 & 1.56 & 1.60 & $1.57 \pm 0.35$ & 0.658 & 0.859 \\
\hline
\end{tabular}

SE - standard error; L - linear; Q - quadratic.

$\mathrm{DM}$ - dry matter; WG - weight gain.

Table 4 - Final body measurements $(\mathrm{cm})$ of dairy feeder calves receiving starter concentrate with addition of crude glycerin

\begin{tabular}{lcccccccc}
\hline \multirow{2}{*}{ Variable } & \multicolumn{4}{c}{ Crude glycerin $\left(\mathrm{g} \mathrm{kg}^{-1} \mathrm{DM}\right)$} & & \multicolumn{3}{c}{$\mathrm{P}$-value } \\
\cline { 2 - 5 } & 0 & 80 & 160 & 240 & & $\mathrm{C}$ & $\mathrm{A}$ & $\mathrm{CG} \times \mathrm{A}$ \\
\hline Thoracic girth & 94.29 & 94.71 & 95.14 & 98.81 & $95.7 \pm 5.25$ & 0.012 & 0.001 & 0.999 \\
Hip width & 23.50 & 24.07 & 24.29 & 24.50 & $24.1 \pm 1.54$ & 0.020 & 0.001 & 0.999 \\
Hip height & 88.60 & 89.86 & 88.77 & 89.00 & $89.1 \pm 3.80$ & 0.588 & 0.0001 & 0.999 \\
Shoulder height & 84.94 & 84.63 & 84.93 & 84.53 & $84.8 \pm 6.12$ & 0.478 & 0.001 & 0.968 \\
Body length & 74.29 & 76.93 & 76.00 & 75.00 & $75.6 \pm 6.08$ & 0.715 & 0.001 & 0.650 \\
\hline
\end{tabular}

SE - standard error; CG - crude glycerin; A - age.

Regression equations for inclusion level of crude glycerin: Thoracic girth $\left(\hat{\mathrm{Y}}=85.9859+0.00661 \mathrm{x}, \mathrm{R}^{2}=0.60\right) ;$ Hip width $\left(\hat{\mathrm{Y}}=21.07+0.0252 \mathrm{x}, \mathrm{R}^{2}=0.66\right)$. 
The omasum weight was not affected $(\mathrm{P}>0.05)$ by including crude glycerin in the concentrate when expressed in absolute weight, adjusted to the BW, or EBW; however, when it was fitted to the total stomach weight, it decreased linearly $(\mathrm{P}<0.05)$ by 0.002 percentage points for each gram of crude glycerin included. The abomasum weight increased linearly $(\mathrm{P}<0.01)$ when expressed in absolute weight, adjusted for BW, and EBW. The weight of the compartments together $(\mathrm{RROA})$ was not influenced $(\mathrm{P}>0.05)$ by adding crude glycerin to the concentrate in any of the ways it was expressed. No effect was observed $(\mathrm{P}>0.05)$ on the rumen papillae height and width (Table 5).

An increase was observed as the calf aged in the absolute weight, weight adjusted to BW, EBW of the stomach (RROA), its compartments (rumen-reticulum, omasum, and abomasum), and the rumen-reticulum volume. Although the measurements of the reference animals were not assessed statistically with the experimental calves fed crude glycerin in the concentrate, when the proportion of the individual compartments was analyzed in relation to

Table 5 - Morphometric measurements of the upper digestive tract of dairy feeder calves receiving starter concentrate with addition of crude glycerin

\begin{tabular}{|c|c|c|c|c|c|c|c|c|}
\hline \multirow{2}{*}{ Variable } & \multirow{2}{*}{ RA } & \multicolumn{4}{|c|}{ Crude glycerin ( $\left.\mathrm{g} \mathrm{kg}^{-1} \mathrm{DM}\right)$} & \multirow{2}{*}{ Mean $\pm \mathrm{SE}$} & \multicolumn{2}{|c|}{ P-value } \\
\hline & & 0 & 80 & 160 & 240 & & $\mathrm{~L}$ & Q \\
\hline \multicolumn{9}{|c|}{ Rumen-reticulum } \\
\hline $\mathrm{kg}$ & 0.185 & 1.53 & 1.47 & 1.59 & 1.80 & $1.59 \pm 0.33$ & 0.121 & 0.118 \\
\hline $\mathrm{kg} / 100 \mathrm{~kg} \mathrm{BW}^{1}$ & 0.502 & 2.08 & 1.96 & 2.16 & 2.35 & $2.14 \pm 0.18$ & 0.002 & 0.754 \\
\hline $\mathrm{kg} / 100 \mathrm{~kg} \mathrm{EBW}{ }^{2}$ & 0.539 & 2.36 & 2.16 & 2.42 & 2.55 & $2.36 \pm 0.21$ & 0.022 & 0.754 \\
\hline Total stomach, $\mathrm{kg} / 100 \mathrm{~kg}^{3}$ & 40.07 & 64.39 & 63.08 & 65.84 & 67.47 & $65.19 \pm 2.66$ & 0.012 & 0.754 \\
\hline Volume, L & 1.38 & 12.54 & 14.84 & 15.07 & 12.18 & $13.65 \pm 1.38$ & 0.712 & 0.754 \\
\hline \multicolumn{9}{|c|}{ Omasum } \\
\hline $\mathrm{kg}$ & 0.061 & 0.44 & 0.43 & 0.40 & 0.37 & $0.41 \pm 0.13$ & 0.269 & 0.543 \\
\hline kg/100 kg BW & 0.168 & 0.62 & 0.56 & 0.55 & 0.50 & $0.55 \pm 0.15$ & 0.156 & 0.372 \\
\hline $\mathrm{kg} / 100 \mathrm{~kg}$ EBW & 0.181 & 0.70 & 0.62 & 0.61 & 0.54 & $0.62 \pm 0.17$ & 0.097 & 0.911 \\
\hline Total stomach, kg/100 kg ${ }^{4}$ & 13.50 & 19.25 & 17.68 & 16.35 & 14.40 & $16.92 \pm 3.66$ & 0.016 & 0.894 \\
\hline \multicolumn{9}{|c|}{ Abomasum } \\
\hline $\mathrm{kg}^{5}$ & 0.206 & 0.38 & 0.44 & 0.41 & 0.45 & $0.42 \pm 0.03$ & 0.001 & 0.754 \\
\hline $\mathrm{kg} / 100 \mathrm{~kg} \mathrm{BW}^{6}$ & 0.572 & 0.53 & 0.59 & 0.57 & 0.61 & $0.57 \pm 0.03$ & 0.001 & 0.754 \\
\hline $\mathrm{kg} / 100 \mathrm{~kg} \mathrm{EBW}^{7}$ & 0.616 & 0.60 & 0.65 & 0.64 & 0.67 & $0.64 \pm 0.09$ & 0.003 & 0.714 \\
\hline Total stomach, kg/100 kg & 46.43 & 16.36 & 19.24 & 17.81 & 18.13 & $17.89 \pm 1.81$ & 0.217 & 0.754 \\
\hline \multicolumn{9}{|c|}{ Sum of compartments } \\
\hline $\mathrm{kg}$ & 0.453 & 2.36 & 2.34 & 2.40 & 2.62 & $2.43 \pm 0.40$ & 0.215 & 0.447 \\
\hline kg/100 kg BW & 1.242 & 3.22 & 3.11 & 3.28 & 3.46 & $3.27 \pm 0.45$ & 0.241 & 0.403 \\
\hline kg/100 kg EBW & 1.336 & 3.66 & 3.42 & 3.68 & 3.76 & $3.63 \pm 0.49$ & 0.514 & 0.388 \\
\hline Papillae height, mm & - & 0.81 & 0.82 & 0.81 & 0.95 & $0.85 \pm 0.19$ & 0.238 & 0.368 \\
\hline Papillae width, mm & - & 0.21 & 0.21 & 0.21 & 0.21 & $0.21 \pm 0.05$ & 0.987 & 0.918 \\
\hline
\end{tabular}

SE - standard error; L - linear; Q - quadratic; RA - reference animals $(n=7)$.

BW - body weight; EBW - empty body weight.

Rumen-reticulum $\left({ }^{1} \hat{\mathrm{Y}}=1.985+0.0013 \mathrm{x}, \mathrm{R}^{2}=0.30 ;{ }^{2} \hat{\mathrm{Y}}=2.245+0.0011 \mathrm{x}, \mathrm{R}^{2}=0.18 ;{ }^{3} \hat{\mathrm{Y}}=63.40+0.015 \mathrm{x}, \mathrm{R}^{2}=0.22\right) ;$ Omasum $\left({ }^{4} \hat{\mathrm{Y}}=19.30-0.0020 \mathrm{x}, \mathrm{R}^{2}=0.20\right) ;$ Abomasum $\left({ }^{5} \hat{Y}=0.389+0.00025 x, R^{2}=0.42 ;{ }^{6} \hat{Y}=0.538+0.00032 x, R^{2}=0.45 ;{ }^{7} \hat{Y}=0.610+0.00024 x, R^{2}=0.30\right)$.

Table 6 - Blood indicators of dairy feeder calves receiving starter concentrate with addition of crude glycerin

\begin{tabular}{|c|c|c|c|c|c|c|c|c|c|}
\hline \multirow{2}{*}{ Variable } & \multirow{2}{*}{ RA } & \multicolumn{4}{|c|}{ Crude glycerin $\left(\mathrm{g} \mathrm{kg}^{-1} \mathrm{DM}\right)$} & \multirow{2}{*}{ Mean $\pm \mathrm{SE}$} & \multicolumn{3}{|c|}{ P-value } \\
\hline & & 0 & 80 & 160 & 240 & & $\mathrm{CG}$ & $\mathrm{C}$ & $\mathrm{CG} \times \mathrm{C}$ \\
\hline $\mathrm{CR}, \mathrm{mg} \mathrm{dL}^{-1}$ & 1.18 & 1.44 & 1.24 & 1.22 & 1.29 & $1.29 \pm 0.30$ & 0.08 & 0.001 & 0.06 \\
\hline Alb, $\mathrm{g} \mathrm{dL}^{-1}$ & 3.00 & 2.41 & 2.47 & 2.91 & 2.56 & $2.58 \pm 0.66$ & 0.06 & 0.051 & 0.008 \\
\hline $\mathrm{TP}, \mathrm{g} \mathrm{dL}-1$ & 7.91 & 6.78 & 6.66 & 6.91 & 6.30 & $6.66 \pm 1.87$ & 0.938 & 0.001 & 0.601 \\
\hline Glb, $\mathrm{g} \mathrm{dL}^{-1}$ & 4.91 & 4.39 & 4.20 & 3.99 & 3.74 & $4.08 \pm 1.90$ & 0.864 & 0.001 & 0.960 \\
\hline GGT, IU L ${ }^{-1}$ & 407.7 & 101.4 & 154.2 & 644.5 & 192.1 & $273.5 \pm 0.45$ & 0.864 & 0.001 & 0.960 \\
\hline AST, IU L L ${ }^{-1}$ & 89.36 & 50.88 & 54.65 & 51.67 & 51.13 & $52.06 \pm 14.07$ & 0.733 & 0.001 & 0.674 \\
\hline ALP, IU L L & - & 180.1 & 226.4 & 231.8 & 206.9 & $211.30 \pm 83.35$ & 0.184 & 0.061 & 0.741 \\
\hline Chol, $\mathrm{mg} \mathrm{dL}^{-1}$ & 63.77 & 82.77 & 66.79 & 72.40 & 56.28 & $69.56 \pm 25.28$ & 0.881 & 0.586 & 0.06 \\
\hline BUN, mg dL $\mathrm{dL}^{-1}$ & 26.94 & 24.85 & 22.83 & 23.96 & 22.30 & $23.49 \pm 8.1$ & 0.740 & 0.257 & 0.739 \\
\hline
\end{tabular}

$\mathrm{SE}$ - standard error; CG - crude glycerin; $\mathrm{C}$ - collection; RA - reference animals $(\mathrm{n}=7)$.

ALP - alkaline phosphatase; chol - cholesterol; BUN - blood urea nitrogen.

Regression equations for the effect of collection $\left(1,2\right.$, and 3): $\mathrm{CR}-$ creatinine $\left(\hat{\mathrm{Y}}=1.93-0.32 \mathrm{x}, \mathrm{R}^{2}=0.83\right) ; \mathrm{Alb}-\mathrm{albumin}\left(\hat{\mathrm{Y}}=3.4-0.42 \mathrm{x}, \mathrm{R}^{2}=0.86\right)$; $\mathrm{TP}-$ total protein $\left(\hat{\mathrm{Y}}=10.77-2.15 \mathrm{x}, \mathrm{R}^{2}=0.83\right) ; \mathrm{Glb}-\operatorname{globulin}\left(\hat{\mathrm{Y}}=8.428-2.23 \mathrm{x}, \mathrm{R}^{2}=0.79\right) ; \mathrm{GGT}-$ gamma glutamyl transferase $\left(\hat{\mathrm{Y}}=2.60-0.48 \mathrm{x}, \mathrm{R}^{2}=0.86\right) ; \mathrm{AST}-\operatorname{aspartate}$ aminotransferase $\left(\hat{\mathrm{Y}}=-0.32+26.01 \mathrm{x}, \mathrm{R}^{2}=0.79\right)$. 
the total stomach weight (Table 5), a 27.4\% increase was observed in the rumen-reticulum of the calves fed concentrate with $240 \mathrm{~g} \mathrm{~kg}^{-1}$ crude glycerin. The proportion of the abomasum in relation to the total stomach decreased by $30.1,27.2,28.6$, and $28.3 \%$, respectively, for the treatments with $0,80,160$, and $240 \mathrm{~g} \mathrm{~kg}^{-1}$ crude glycerin compared with the reference animals.

Including crude glycerin in the concentrate did not change $(\mathrm{P}>0.05)$ the blood parameters (Table 6). However, as the animals grew older, the concentrations of creatinine, albumin, total proteins, globulins, and gamma glutamyl transferase (GGT) decreased linearly $(\mathrm{P}<0.01)$, while aspartate aminotransferase (AST) values increased linearly. The plasma concentrations of urea, cholesterol, and alkaline phosphatase (AP) were not affected $(\mathrm{P}>0.05)$ by including crude glycerin in the concentrate or by calf age.

Including crude glycerin in the concentrate did not influence $(\mathrm{P}>0.05)$ the feeder calf carcass traits (Table 7$)$. The muscle percentages in the carcass of the animals that received concentrate with $0,80,160$, and $240 \mathrm{~g} \mathrm{~kg}^{-1}$ crude glycerin added, when compared with the reference animals, presented an additional $4.5,3.6,4.3$, and $2.5 \%$, respectively. The percentage of bone in the carcass decreased on average by $6.14 \%$, and the fat percentage in the carcasses

Table 7 - Quantitative carcass characteristics of calves fed concentrates containing crude glycerin

\begin{tabular}{|c|c|c|c|c|c|c|c|c|}
\hline \multirow{2}{*}{ Variable } & \multirow{2}{*}{ RA } & \multicolumn{4}{|c|}{ Crude glycerin $\left(\mathrm{g} \mathrm{kg}^{-1} \mathrm{DM}\right)$} & \multirow{2}{*}{ Mean \pm SE } & \multicolumn{2}{|c|}{ P-value } \\
\hline & & 0 & 80 & 160 & 240 & & $\mathrm{~L}$ & Q \\
\hline EBW, kg & 33.91 & 64.25 & 67.52 & 64.87 & 68.80 & $66.36 \pm 8.36$ & 0.443 & 0.713 \\
\hline $\mathrm{ACG}, \mathrm{kg} \mathrm{d}^{-1}$ & - & 0.538 & 0.587 & 0.560 & 0.606 & $0.573 \pm 0.13$ & 0.723 & 0.646 \\
\hline HCW, kg & 19.92 & 38.16 & 39.74 & 37.64 & 40.14 & $38.92 \pm 5.3$ & 0.670 & 0.870 \\
\hline $\mathrm{HCY}, \mathrm{kg} / 100 \mathrm{~kg} \mathrm{BW}$ & 54.52 & 52.18 & 53.32 & 51.92 & 53.60 & $52.76 \pm 2.69$ & 0.540 & 0.797 \\
\hline HCY, kg/100 kg EBW & 58.57 & 59.24 & 58.67 & 57.91 & 58.40 & $58.55 \pm 2.04$ & 0.350 & 0.507 \\
\hline $\mathrm{CCW}, \mathrm{kg}$ & 19.09 & 36.33 & 38.60 & 36.21 & 38.39 & $37.38 \pm 5.22$ & 0.671 & 0.754 \\
\hline CCY, kg/100 kg BW & 52.31 & 49.74 & 51.80 & 49.95 & 51.17 & $50.66 \pm 2.18$ & 0.515 & 0.618 \\
\hline $\mathrm{CCY}, \mathrm{kg} / 100 \mathrm{~kg}$ EBW & 56.21 & 56.52 & 57.01 & 55.71 & 55.74 & $56.24 \pm 1.71$ & 0.219 & 0.730 \\
\hline LLA, cm & 12.11 & 21.37 & 22.22 & 21.78 & 20.7 & $20.70 \pm 2.08$ & 0.491 & 0.754 \\
\hline Muscle, kg/100 kg CC & 64.7 & 69.19 & 68.27 & 68.99 & 67.21 & $67.21 \pm 3.54$ & 0.390 & 0.7548 \\
\hline Bone, kg/100 kg CC & 26.1 & 20.30 & 19.26 & 20.01 & 20.27 & $20.27 \pm 2.09$ & 0.851 & 0.4177 \\
\hline Fat, $\mathrm{kg} / 100 \mathrm{~kg} \mathrm{CC}$ & 6.0 & 9.26 & 11.60 & 9.85 & 11.30 & $10.50 \pm 2.73$ & 0.349 & 0.6762 \\
\hline
\end{tabular}

SE - standard error; L - linear; Q - quadratic; RA - reference animals $(n=7)$.

EBW - empty body weight; ACG - average carcass gain; HCW - hot carcass weight; CCW - cold carcass weight; HCY - hot carcass yield; CCY - cold carcass yield; LLA - longissimus lumborum area; CC - cold carcass.

Table 8 - Cuts of the pistol cut of calves fed concentrate containing crude glycerin

\begin{tabular}{|c|c|c|c|c|c|c|c|c|}
\hline \multirow{2}{*}{ Variable } & \multirow{2}{*}{ RA } & \multicolumn{4}{|c|}{ Crude glycerin $\left(\mathrm{g} \mathrm{kg}^{-1} \mathrm{DM}\right)$} & \multirow{2}{*}{ Mean $\pm \mathrm{SE}$} & \multicolumn{2}{|c|}{ P-value } \\
\hline & & 0 & 80 & 160 & 240 & & $\mathrm{~L}$ & Q \\
\hline Rump uk trim, kg & 0.20 & 0.50 & 0.56 & 0.51 & 0.54 & $0.53 \pm 0.07$ & 0.591 & 0.825 \\
\hline Rump uk trim, kg/100 kg BW & 4.10 & 5.35 & 5.67 & 5.39 & 5.47 & $5.47 \pm 0.34$ & 0.908 & 0.642 \\
\hline Tail of rump, kg & 0.05 & 0.15 & 0.15 & 0.13 & 0.15 & $0.15 \pm 0.01$ & 0.923 & 0.947 \\
\hline Tail of rump, $\mathrm{kg} / 100 \mathrm{~kg} \mathrm{BW}$ & 0.99 & 1.57 & 1.58 & 1.43 & 1.54 & $1.53 \pm 0.25$ & 0.560 & 0.731 \\
\hline Striploin, $\mathrm{kg}$ & 0.37 & 0.88 & 1.01 & 0.93 & 0.97 & $0.95 \pm 0.12$ & 0.369 & 0.398 \\
\hline Striploin, kg/100 kg BW & 7.52 & 9.41 & 10.09 & 9.81 & 9.82 & $9.78 \pm 0.87$ & 0.519 & 0.488 \\
\hline Eye of round, $\mathrm{kg}$ & 0.12 & 0.28 & 0.31 & 0.29 & 0.30 & $0.30 \pm 0.04$ & 0.573 & 0.814 \\
\hline Eye of round, $\mathrm{kg} / 100 \mathrm{~kg} \mathrm{BW}$ & 2.51 & 3.02 & 3.10 & 3.03 & 3.07 & $3.06 \pm 0.31$ & 0.862 & 0.970 \\
\hline Rump cap, $\mathrm{kg}$ & 0.06 & 0.22 & 0.21 & 0.19 & 0.24 & $0.22 \pm 0.04$ & 0.681 & 0.819 \\
\hline Rump cap, kg/100 kg BW & 1.25 & 2.39 & 2.16 & 2.08 & 2.40 & $2.26 \pm 0.49$ & 0.960 & 0.337 \\
\hline Inside, $\mathrm{kg}$ & 0.57 & 1.25 & 1.22 & 1.26 & 1.31 & $1.26 \pm 0.16$ & 0.410 & 0.682 \\
\hline Inside, $\mathrm{kg} / 100 \mathrm{~kg} \mathrm{BW}$ & 11.5 & 13.47 & 12.30 & 13.38 & 13.33 & $13.12 \pm 0.88$ & 0.667 & 0.220 \\
\hline Outside, $\mathrm{kg}$ & 0.27 & 0.60 & 0.84 & 0.64 & 0.66 & $0.69 \pm 0.13$ & 0.938 & 0.995 \\
\hline Outside, kg/100 kg BW & 5.50 & 6.43 & 8.14 & 6.72 & 6.71 & $7.00 \pm 1.8$ & 0.857 & 0.446 \\
\hline Shank, kg & 0.43 & 0.78 & 0.82 & 0.76 & 0.80 & $0.79 \pm 0.13$ & 0.984 & 0.951 \\
\hline Shank, kg/100 kg BW & 8.69 & 8.42 & 8.15 & 8.10 & 8.19 & $8.22 \pm 0.23$ & 0.064 & 0.176 \\
\hline Cuberoll, $\mathrm{kg}$ & 0.05 & 0.11 & 0.12 & 0.13 & 0.12 & $0.12 \pm 0.03$ & 0.341 & 0.397 \\
\hline Cuberoll, kg/100 kg BW & 0.99 & 1.16 & 1.23 & 1.36 & 1.24 & $1.25 \pm 0.10$ & 0.058 & 0.164 \\
\hline Tenderloin, kg & 0.14 & 0.36 & 0.38 & 0.34 & 0.35 & $0.36 \pm 0.06$ & 0.513 & 0.771 \\
\hline Tenderloin, kg/100 kg BW & 2.89 & 3.84 & 3.77 & 3.61 & 3.56 & $3.70 \pm 0.11$ & 0.001 & 0.754 \\
\hline Knuckle, kg & 0.43 & 0.86 & 0.87 & 0.80 & 0.87 & $0.85 \pm 0.12$ & 0.785 & 0.917 \\
\hline Knuckle, kg/100 kg BW & 8.84 & 9.22 & 8.82 & 8.47 & 8.85 & $8.84 \pm 0.54$ & 0.119 & 0.641 \\
\hline
\end{tabular}

SE - standard error; L - linear; $\mathrm{Q}$ - quadratic; RA - reference animals $(\mathrm{n}=7)$.

Tenderloin $\left(\hat{\mathrm{Y}}=3.848-0.0013 \mathrm{x}, \mathrm{R}^{2}=0.53\right)$. 
increased by $3.2,5.6,3.9$, and $5.3 \%$, respectively, for the treatments with $0,80,160$, and $240 \mathrm{~g} \mathrm{~kg}^{-1}$ crude glycerin. The secondary cuts of the pistol cut - rump uk trim, tail of rump, striploin, eye of round, rump cap, inside, outside, shank, cuberoll, tenderloin, and knuckle - were not influenced $(\mathrm{P}>0.05)$ by including crude glycerin in the concentrate, when expressed in $\mathrm{kg}$ (Table 8). When the cuts were assessed for their relative participation in the special hindquarter cuts, tenderloin decreased linearly $(\mathrm{P}<0.01)$ with the inclusion of crude glycerin in the concentrate.

\section{Discussion}

Including crude glycerin in the concentrate led to a significant increase in DMI, perhaps because crude glycerin is viscose, hygroscopic, and has a relatively sweet flavor (Donkin and Doane, 2007), characteristics that increase the palatability of the diets. Furthermore, concentrates mixed with crude glycerin have a thick, moist texture with wellaggregated components that may have contributed to higher intake by the calves. However, previous studies did not reveal an effect on DMI $(0.741 \mathrm{~kg})$ when 30 and $60 \mathrm{~g} \mathrm{~kg}^{-1}$ corn were replaced with crude glycerin in pellet concentrate for unweaned dairy calves (Chester-Jones et al., 2010).

Regarding the increase in DMI as a function of age, its response is commonly observed in feeder calves in the unweaned phase, as reported in other studies (Bach et al., 2007; Khan et al., 2008; Lee et al., 2008; Bach et al., 2013), as a result of animal growth and because the intake of a fixed quantity of milk does not meet the entire maintenance and weight-gain requirements of the animals.

Research is being carried out to speed solid food intake so that animals can be weaned younger. Because the DMI was greater due to the addition of crude glycerin, the calves could be weaned earlier, which would result in lower feeding costs because concentrate is cheaper than milk or dried milk and also stimulates rumen development and contributes to supplying nutrients to animals (Baldwin et al., 2004; Suárez et al., 2006; Khan et al., 2007). Considering the intake of $700 \mathrm{~g} \mathrm{day}^{-1}$ concentrate (Quigley III, 1996; Campos and Lizieire, 2000) to carry out weaning, the calves that received concentrate with $240,160,80$, and $0 \mathrm{~g} \mathrm{~kg}^{-1}$ crude glycerin included could have been weaned at $40,42,44$, and 46 days of age, respectively (Figure 1). This difference is significant when expenses with feeding and labor, milk commercialization, and animal performance are considered (Roth et al., 2009; Hulbert et al., 2011).

The greater DMI observed when crude glycerin was added to the concentrate favored the performance of the calves. However, regardless of the treatment, all animals presented considerable ideal gains (over $0.600 \mathrm{~g} \mathrm{day}^{-1}$ ) for the unweaned phase (Hoffman, 1997), allowing the calves to reach twice their initial weight at weaning (ChesterJones et al., 2010). Drackley (2008) assessed milk replacer with $150 \mathrm{~g} \mathrm{~kg}^{-1}$ crude glycerin for Friesian calves, which reduced the percentage of lactose, but did not affect the ADG, body measurements, or animal health.

The increase in thoracic girth in the calves that received concentrate with the addition of $240 \mathrm{~g} \mathrm{~kg}^{-1}$ crude glycerin was $17 \%$ greater than that of the calves fed the standard concentrate. The thoracic girth is highly correlated with the animal live weight (Heinrichs et al., 1992; Ozkaya and Bozkurt, 2009) and the increase in this measurement in the present study may be attributed to the greater performance of the animals receiving crude glycerin (Table 2). All the body measurements recorded are considered adequate and similar to data obtained in other studies with calves in the same phase (Blome et al., 2003; Lesmeister and Heinrichs, 2005; Kehoe et al., 2007; Khan et al., 2007).

The data show the positive effect of including crude glycerin in starter concentrate on rumen-reticulum development, which was probably due to the higher concentrate intake, which is considered the main stimulant to develop the rumen by the end product of its fermentation (Harrison et al., 1960; Lesmeister and Heinrichs, 2004; Coverdale et al., 2004; Khan et al., 2008; Boyd et al., 2013). The rumen-reticulum weight and volume increased ninefold in eight weeks in the animals fed concentrate with $240 \mathrm{~g} \mathrm{~kg}^{-1}$ crude glycerin, compared with the reference animals. These results show the importance of supplying solid foods for the rumen-reticulum muscular development when early weaning is desired and given the potential of crude glycerin as an alternative feedstuff to corn in calf starter concentrate. Regarding the abomasum weight, the highest values observed for this compartment in the treatments with crude glycerin were associated with greater DMI. At birth (reference), the abomasum occupied a greater proportion, but with the ingestion of solid feedstuffs the rumen-reticulum occupied a greater proportional weight, in agreement with other research with calves of equivalent ages (Khan et al., 2007; Bittar et al., 2009). Papilla development is linked to the presence of solid feedstuffs in the rumen, short-chain fatty acid production resulting from fermentation, and the stimulus caused by the feedstuffs (Sander et al., 1959; Tamate et al., 1962).

Although the blood creatinine concentration decreased as calf age increased, it was in the normal range for calves from 1 to 8 weeks (Klinkon and Ježek, 2012). A decrease in creatinine concentration with age is an indicator of normal and gradual increase in the kidney function capacity of the 
calves (Benesi et al., 2003; Mohri et al., 2007). Similar performance for creatinine concentrations in relation to age was reported by Khan et al. (2007), Mohri et al. (2007), and Lee et al. (2008). Creatinine levels are not deeply affected by diet (Klinkon and Ježek, 2012) and are normally used as auxiliary reference to diagnose variations in blood urea. The plasma urea levels were not altered by including crude glycerin and remained within the levels observed in other studies with calves at equivalent age (Knowles et al., 2000; Benesi et al., 2003; Klinkon and Ježek, 2012), emphasizing that the kidney functions of the calves were normal.

The total protein and globulin concentrations varied with age, but were within the range considered normal for physiological standards (Fagliari et al., 1998; Leal et al., 2003). The total protein, albumin, and globulin concentrations are important in diagnosing several diseases and disorders in the liver function (Klinkon and Ježek, 2012). The changes in the serum concentrations of total protein and globulin performed similarly as the animal age increased and were compatible with those observed by Mohri et al. (2007) for calves up to eight weeks of age. The variations in albumin concentrations with age were only observed in the animals with $240 \mathrm{~g} \mathrm{~kg}^{-1}$ crude glycerin, but remained within the reference interval for calves (2.7 to $3.9 \mathrm{~g} \mathrm{dL}^{-1}$ ) (Knowles et al., 2000).

Serum GGT activities in the calves decreased as age increased, reaching stable values reported for eight-week old calves with normal hepatic function (Klinkon and Ježek, 2012). After colostrum is ingested, the GGT enzyme activity increases because it is absorbed through the intestinal wall and therefore its use for the assessment of the liver function is limited in the first days of life (Braun et al., 1982), but it can be used as an indirect indicator of passive immunity transference to calves (Feitosa et al., 2007), together with the total serum protein and globulin concentrations (Mohri et al., 2007). In spite of the greater serum GGT activity in the first collection (fifth day of life) recorded for the calves on the treatment with $160 \mathrm{~g} \mathrm{~kg}^{-1}$ crude glycerin, all the treatments received an adequate quantity $\left(848.8 \mathrm{IU} \mathrm{L}^{-1}\right)$ of colostrum, considering the minimum value of $200 \mathrm{IU} \mathrm{L}^{-1}$ reported by Perino et al. (1993) for correct colostrum administration.

Aspartate aminotransferase activity varied with age, which is in line with Mohri et al. (2007), who observed high values 24 to $48 \mathrm{~h}$ after birth and an increase in AST activity from 14 to 84 days of age. In the first and second collections, the AST activity varied within the reference values established by Klinkon and Ježek (2012), indicating no liver alterations. The activity of the AP enzyme, which can also be used to indicate liver damage (González and Scheffer, 2003), ranged within the normal values, showing that the calves had no liver problem that might be related to an increase in AST activity. The cholesterol concentrations observed were in agreement with those reported by Pogliani and Birgel Junior (2007) for unweaned calves up to three months of age and were not influenced by age or treatment, which can be attributed to the constant offer of milk throughout the experimental period.

Generally, the similarity amongst treatments for the variables related to the carcass traits can be attributed to the similar weight conditions under which they were slaughtered (Kuss et al., 2005). In studies on finishing steers (Mach et al., 2009; Bartoň et al., 2013) and calves (Ramos and Kerley, 2012), similar carcass traits were described when compared with animals fed a diet without crude glycerin and diets with up to $200 \mathrm{~g} \mathrm{~kg}^{-1}$ crude glycerin. The values observed for carcass weight and yield agree with the results obtained by Brown et al. (2005) for calves slaughtered at the same weight and age as in the present study. In Brazil, there is no expressive consumption of veal such as the animals slaughtered in the present study due to economic and cultural questions. There are few studies on carcass characteristics and the meat of these animals, which hinders the establishment of a standard for this product in tropical conditions, requiring more research on the subject.

When the tissue percentages of the reference animals are considered, the decrease in the proportion of bone with an increase in age was due to the increase in the proportion of muscle and fat. This is because tissue percentages in the carcass are relative units, and an increase or decrease in the participation of a tissue in the total results in a decrease or increase in the other. Although the calves were in full development, this clearly demonstrated the changes in the tissue composition of the animals with increasing age. The higher meat yield of calves slaughtered at eight weeks makes them more attractive for commercialization.

\section{Conclusions}

Crude glycerin at up to $240 \mathrm{~g} \mathrm{~kg}^{-1}$ of the dry matter of the concentrate can be added as an alternative to the use of corn in calf feeding because it results in higher concentrate intake, average daily weight gain, and rumen development without affecting the quality of the carcass or the health of the animals.

\section{Acknowledgments}

The authors would like to thank Cooperativa Agroindustrial dos Produtores Rurais do Sudoeste Goiano (COMIGO), GRANOL S.A., and the National Council 
for Scientific and Technological Development (Conselho Nacional de Desenvolvimento Científico e Tecnológico $\mathrm{CNPq}$ ) for their financial support.

\section{References}

AOAC - Association of Official Analytical Chemists. 1995. Official methods of analysis. 16th ed. AOAC International, Arlington, VA.

Bach, A.; Giménez, A.; Jaristi, J. L. and Ahedo, J. 2007. Effects of physical form of a starter for dairy replacement calves on feed intake and performance. Journal of Dairy Science 90:3028-3033.

Bach, A.; Terré, M. and Pinto, A. 2013. Performance and health responses of dairy calves offered different milk replacer allowances. Journal of Dairy Science 96:7790-7797.

Baldwin, R. L. V.; McLeod, K. R.; Klotz, J. L. and Heitmann, R. N. 2004. rumen development, intestinal growth and hepatic metabolism in the pre-and postweaning ruminant. Journal of Dairy Science 87(Supp.):E55-E65.

Bartoň, L.; Bureš, D.; Homolka, P.; Jančík, F.; Marounek, M. and Řehák, D. 2013. Effects of long-term feeding of crude glycerine on performance, carcass traits, meat quality, and blood and rumen metabolites of finishing bulls. Livestock Science 155:53-59.

Benesi, F. J.; Leal, M. L. R.; Lisbôa, J. A. N.; Coelho, C. S. and Mirandola, R. M. S. 2003. Parâmetros bioquímicos para avaliação da função hepática em bezerras sadias, da raça holandesa, no primeiro mês de vida. Ciência Animal 33:311-317.

Bittar, C. M. M.; Ferreira, L. S.; Santos, F. A. P. and Zopollatto, M. 2009. Desempenho e desenvolvimento do trato digestório superior de bezerros leiteiros alimentados com concentrados de diferentes formas físicas. Revista Brasileira de Zootecnia 38:1561-1567.

Boyd, J.; Bernard, J. K. and West, J. W. 2013. Effects of feeding different amounts of supplemental glycerol on ruminal environment and digestibility of lactating dairy cows. Journal of Dairy Science 96:470-476.

Blome, R. M.; Drackley, J. K.; McKeith, F. K.; Hutjens, M. F. and McCoy, G. C. 2003. Growth, nutrient utilization, and body composition of dairy calves fed milk replacers containing different amounts of protein. Journal of Animal Science 81:1641-1655.

Braun, D. P.; Tainturier, D.; Laugter, C.; Benard, P.; Thouvenot, J. P. and Rico, A. G. 1982. Early variations of blood plasma gammaglutamyl transferase in Newborn calves - A test of colostrum intake. Journal Dairy Science 65:2178-2181.

Brown, E. G.; Vandehaar, M. J.; Daniels, K. M.; Liesman, J. S.; Chapin, L. T.; Keisler, D. H. and Weber Nielsen, M. S. 2005. Effect of increasing energy and protein intake on body growth and carcass composition of heifer calves. Journal of Dairy Science 88:585-594.

Campos, O. F. and Lizieire, R. S. 2000. Produção do bezerrão. 21p. Embrapa Gado de Leite, Juiz de Fora, MG. (Embrapa Gado de Leite/Circular Técnica, 58).

Coverdale, J. A.; Tyler, H. D.; Quigley III, J. D. and Brumm, J. A. 2004. Effect of various levels of forage and form of diet on rumen development and growth in calves. Journal of Dairy Science 87:2554-2562.

Chester-Jones, H.; Ziegler, D.; Timmerman, D.; Golombeski, G. and Raeth-Knight, M. 2010. Use of glycerol as a corn replacement in calf starter diets. Agricultural Utilization Research Institute. Project $\mathrm{N}^{\circ}$ AIC044. Waseca, MN, USA.

Donkin, S. S. 2008. Glycerol from biodiesel production: the new corn for dairy cattle. Revista Brasileira de Zootecnia 37:280-286.

Donkin, S. S. and Doane, P. 2007. Glycerol as a feed ingredient in dairy rations. p.97-103. In: Proceedings of the Tri-State Dairy Nutrition Conference, Fort Wayne, Indiana, USA.
Drackley, J. K. 2008. Opportunities for glycerol use in dairy diets. p.113-118. In: Proceedings of the 4th State Dairy Nutrition and Management Conference, Dubuque. SDN, Dubuque.

Ebert, R. A.; Willis, G. M. and Drackley, J. K. 2008. Efficacy of glycerol as a replacement for lactose in calf milk replacer. Journal Animal Science 86:68/Journal Dairy Science 91:68 (Abstract 198).

Fagliari, J. J.; Santana, A. E.; Lucas, F. A.; Campos Filho, E. and Curi, P. R. 1998. Constituintes sanguíneos de bovinos recém-nascidos das raças Nelore (Bos indicus) e Holandesa (Bos taurus) e de bubalinos (Bubalus bubalis) da raça Murrah. Arquivo Brasileiro de Medicina Veterinária e Zootecnia 50:253-262.

Feitosa, F. L. F.; Mendes, L. C. N.; Peiró, J. R.; Cadioli, F. A.; Yanaka, R.; Bovino, F.; Féres, F. C. and Perri, S. H. V. 2007. Influência da faixa etária nos valores de enzimas hepáticas e de uréia e creatinina em bezerros holandeses do nascimento até os 365 dias de vida. Ciência Veterinária nos Trópicos 10:54-61.

González, F. H. D. and Scheffer, J. F. S. 2003. Perfil sangüíneo: ferramenta de análise clínica, metabólica e nutricional. p.73-89. In: Anais do 1ำ Simpósio de Patologia Clínica Veterinária da Região Sul do Brasil. González, F. H. D., Campos, R., eds. Universidade Federal do Rio Grande do Sul, Porto Alegre.

Hankins, O. G. and Howe, P. E. 1946. Estimation of the composition of beef carcasses and cuts. Technical Bulletin - USDA, 926. Washington, DC.

Harrison, H. N.; Warner, R. G.; Sander, E. G. and Loosli, J. K. 1960. Changes in the tissue and volume of the stomachs of calves following the removal of dry feed or consumption of inert bulk. Journal of Dairy Science 43:1301-1312.

Heinrichs, A. J.; Rogers, W. O. and Cooper, J.B. 1992. Predicting body weight and wither height in Holstein heifers using body measurements. Journal of Dairy Science 75:3576-3581.

Hoffman, P. C. 1997. Optimum body size of Holstein replacement heifers. Journal of Animal Science 75:836-845.

Hulbert, L. E.; Cobb, C. J.; Carroll, J. A. and Ballou, M. A. 2011. The effects of early weaning on innate immune responses of Holstein calves. Journal of Dairy Science 94:2545-2556.

Kehoe, S. I.; Dechow, C. D. and Heinrichs, A. J. 2007. Effects of weaning age and milk feeding frequency on dairy calf growth, health and rumen parameters. Livestock Science 110:267-272.

Khan, M. A.; Lee, H. J.; Lee, W. S.; Kim, H. S.; Ki, K. S.; Hur, T. Y.; Suh, G. H.; Kang, S. J. and Choi, Y. J. 2007. Structural growth, rumen development, and metabolic and immune responses of Holstein male calves fed milk through step-down and conventional methods Journal of Dairy Science 90:3376-3387.

Khan, M. A.; Lee, H. J.; Lee, W. S.; Kim, H. S.; Kim, S. B.; Park, S. B.; Baek, K. S.; Ha, J. K. and Choi, Y. J. 2008. Starch source evaluation in calf starter: II. Ruminal parameters, rumen development, nutrient digestibilities, and nitrogen utilization in Holstein calves. Journal of Dairy Science 91:1140-1149.

Kuss, F.; Restle, J.; Brondani, I. L.; Pascoal, L. L.; Fernando, L.; Menezes, G.; Pazdiora, R. D. and Freitas, L. S. 2005. Características da carcaça de vacas de descarte de diferentes grupos genéticos terminadas em confinamento com distintos pesos. Revista Brasileira Zootecnia 34:915-925.

Klinkon, M. and Ježek, J. 2012. Values of blood variables in calves. In: A Bird's-Eye View of Veterinary Medicine. Tech. Available at: <http://www.intechopen.com/books/a-bird-s-eyeview-of-veterinary-medicine/values-of-blood-variables-in-calves.> Accessed on: Nov. 5, 2013.

Knowles, T. G.; Edwards, J. E.; Bazeley, K. J.; Brown, S. N.; Butterworth, A.; Warriss, P. D. 2000. Changes in the blood biochemical and haematological profile of neonatal calves with age. Veterinary Record 147:593-598.

Leal, M. L. R.; Benesi, F. J.; Lisbôa, J. A. N.; Coelho, C. S. and Mirandola, R. M. S. 2003. Proteinograma sérico de bezerras sadias, 
da raça holandesa, no primeiro mês pós-nascimento. Brazilian Journal of Veterinary Research and Animal Science 40:138-145.

Lee, H. J.; Khan, M. A.; Lee, W. S.; Kim, H. S.; Ki, K. S.; Kang, S. J.; Hur, T. Y.; Khan, M. S. and Choi, Y. J. 2008. Growth, blood metabolites, and health of holstein calves fed milk replacer containing different amounts of energy and protein. AsianAustralasian Journal of Animal Science 21:198-203.

Lesmeister, K. E. and Heinrichs, A. J. 2004. Effects of corn processing on growth characteristics, rumen development, and rumen parameters in neonatal dairy calves, Journal of Dairy Science 87:3439-3450.

Lesmeister, K. E.; Tozer, P. R. and Heinrichs, A. J. 2004. Development and analysis of a rumen tissue sampling procedure. Journal of Dairy Science 87:1336-1344.

Lesmeister, K. E. and Heinrichs, A. J. 2005. Effects of adding extra molasses to a texturized calf starter on rumen development, growth characteristics, and blood parameters in neonatal dairy calves. Journal of Dairy Science 88:411-418.

Mach, N.; Bach, A. and Devant, M. 2009. Effects of crude glycerin supplementation on performance and meat quality of Holstein bulls fed high-concentrate diets. Journal of Animal Science 87:632-638.

Mohri, M.; Sharifi, K. and Eidi, S. 2007. Hematology and serum biochemistry of Holstein dairy calves: Age related changes and comparison with blood composition in adults. Research in Veterinary Science 83:30-39.

Napoles, G. G. O.; Oltramari, C. E.; Silva, J. T.; Mourão, G. B. and Bittar, C. M. M. 2012. Crude glycerin as a replacement for corn in starter concentrate for dairy calves: Ruminal and blood parameters. Journal Animal Science 90: Suppl. 3/Journal Dairy Science 95: Suppl. 2 (Abstract 285).

NRC - National Research Council. 2001. Nutrient requirement of dairy cattle. 7th rev. ed. National Research Council, National Academy Press, Washington, D.C.

Ozkaya, S. and Bozkurt, Y. 2009. The accuracy of prediction of body weight from body measurements in beef cattle. Archiv Tierzucht 52:371-377.

Perino, L. J.; Sutherland, R. L. and Woollen, N. E. 1993. Serum $\gamma$-glutamiltransferase activity and protein concentration at birth and after suckling in calves with adequate and inadequate passive transfer of immunoglobulin G. American Journal of Veterinary Research 54:56-59.
Pogliani, F. C. and Birgel Junior, E. 2007. Valores de referência do lipidograma de bovinos da raça holandesa, criados no Estado de São Paulo. Brazilian Journal of Veterinary Research and Animal Science 44:373-383.

Quigley III, J. D. 1996. Influence of weaning method on growth, intake and selected blood metabolites in Jersey calves. Journal of Dairy Science 79:2255-2260.

Raeth-Knight, M.; Linn, J.; Larson, R. and Salzer, J. 2009. Impact of glycerol in milk replacer on dairy calf performance. Journal Animal Science 87: E-Suppl. 2/Journal Dairy Science 92: E-Suppl. 1 (Abstract 223).

Rahmat, N.; Abdullah, A. Z. and Mohammed A. R. 2010. Recent progress on innovative and potential technologies for glycerol transformation into fuel additives: a critical review. Renewable and Sustainable Energy Reviews 14:987-1000.

Ramos, M. H. and Kerley, M. S. 2012. Effect of dietary crude glycerol level on ruminal fermentation in continuous culture and growth performance of beef calves. Journal of Animal Science 90:892-899.

Roth, B. A.; Keil, N. M.; Gygax, L. and Hillmann, E. 2009. Influence of weaning method on health status and rumen development in dairy calves. Journal of Dairy Science 92:645-656.

Sander, E. G.; Warner, R. G.; Harrison, H. N. and Loosli, J. K. 1959. The stimulatory effect of sodium butyrate and sodium propionate on the development of rumen mucosa in the young calf. Journal of Dairy Science 42:1600-1605.

Suárez, B. J.; Van Reenen, C. G.; Gerrits, W. J. J.; Stockhofe, N.; Van Vuuren, A. M. and Dijkstra, J. 2006. Effects of supplementing concentrates differing in carbohydrate composition in veal calf diets: II - Rumen development. Journal of Dairy Science 89:4376-4386.

Sniffen, C. J.; O'Connor, J. D.; Van Soest, P. J.; Fox, D. G. and Russell, J. B. 1992. A net carbohydrate and protein system for evaluating cattle diets: II - Carbohydrate and protein availability. Journal of Animal Science 70:35621-3577.

Tamate, H.; McGilliard, A. D.; Jacobson, N. L. and Getty, R. 1962. Effect of various dietaries on the anatomical development of the stomach in the calf. Journal of Dairy Science 45:408-420.

Van Cleef, E. H. C. B.; Ezequiel, J. M. B.; D`Aurea, A. P.; Fávaro, V. R. and Sancanari, J. B. D. 2014. Crude glycerin in diets for feedlot Nellore cattle. Revista Brasileira de Zootecnia 43:86-91.

Werner Omazic, A.; Traven, M.; Roos, S.; Mellgren, E.; Holtenius, K. 2013. Oral rehydration solution with glycerol to dairy calves: effects on fluid balance, metabolism, and intestinal microbiota. Acta Agriculturae Scandinavica Section A 63:47-56. 